\title{
Pairs of Nonsingular Matrices ${ }^{1}$
}

\section{Karl Goldberg}

\section{Institute for Basic Standards, National Bureau of Standards, Washington, D.C.}

\author{
(February 16, 1966)
}

\begin{abstract}
Let $R_{1}$ and $R_{2}$ be $m$ by $n$ matrices of rank $m$. Let $S_{1}$ and $S_{2}$ be $n-m$ by $n$ matrices of rank $n-m$ such that $R_{1} S_{1}^{T}=R_{2} S_{2}^{T}=0$. Then $R_{1} R_{2}^{T}$ is nonsingular if and only if $S_{1} S_{2}^{T}$ is nonsingular, in which case $R_{2}^{T}\left(R_{1} R_{2}^{T}\right)^{-1} R_{1}+\left\{S_{2}^{T}\left(S_{1} S_{2}^{T}\right)^{-1} S_{1}\right\}^{T}$ equals the identity matrix of order $n$.
\end{abstract}

Key Words: Matrices (pairs of nonsingular).

Let $\mathscr{R}_{m n}$ denote the set of $m$ by $n$ matrices of rank $m$, with entries from a field of characteristic 0 . Given $R \epsilon \mathscr{R}_{m n}$ let $\mathscr{N}(R)$ denote the set of $S \epsilon \mathscr{R}_{n-m, n}$ such that $R S^{T}=0$. Note that $S \epsilon \mathscr{N}(R)$ if and only if $R \in \mathscr{N}(S)$.

We assume as known the following results for $R \epsilon \mathscr{R}_{m n}$ and $S \epsilon \mathscr{N}(R):$

LEMMA 1. $\mathrm{XR}=0$ if and only if $\mathrm{X}=0$.

LEMMA 2. $R Y^{T}=0$ if and only if $\mathrm{Y}=\mathrm{ZS}$, for some matrix $\mathrm{Z}$.

The obvious stipulations are that $X, Y$, and $Z$ have $m, n$ and $n-m$ columns respectively, and $Y$ and $Z$ have the same number of rows. Using these lemmas we prove:

THEOREM. If $\mathrm{R}_{1}, \quad \mathrm{R}_{2} \epsilon \mathscr{R}_{\mathrm{mn}}, \quad \mathrm{S}_{1} \in \mathscr{N}\left(\mathrm{R}_{1}\right), \quad \mathrm{S}_{2} \in \mathscr{N}\left(\mathrm{R}_{2}\right)$ then $\mathrm{R}_{1} \mathrm{R}_{2}^{\mathrm{T}}$ is nonsingular if and only if $\mathrm{S}_{1} \mathrm{~S}_{2}^{\mathrm{T}}$ is nonsingular, in which case

$$
\mathbf{R}_{2}^{\mathrm{T}}\left(\mathbf{R}_{1} \mathbf{R}_{2}^{\mathrm{T}}\right)^{-1} \mathbf{R}_{1}+\left\{\mathrm{S}_{2}^{\mathrm{T}}\left(\mathrm{S}_{1} \mathrm{~S}_{2}^{\mathrm{T}}\right)^{-1} \mathrm{~S}_{1}\right\}^{\mathrm{T}}=\mathrm{I}_{n},
$$

where $\mathrm{I}_{\mathrm{n}}$ is the identity matrix of order $\mathrm{n}$.

Suppose $R_{1} R_{2}^{T}$ is singular. Then there exists an $X \neq 0$ such that $X R_{1} R_{2}^{T}=0$.

Let $Y=X R_{1}$. By Lemma $1, Y \neq 0$. We have $R_{2} Y^{T}=R_{2} R_{1}^{T} X^{T}=\left(X R_{1} R_{2}^{T}\right)^{T}=0$.

By Lemma 2, this implies that there exists a $Z$ such that $Y=Z S_{2}$. Clearly $Z \neq 0$. We have $\left(S_{1} S_{2}\right) Z^{T}$ $=S_{1} Y^{T}=S_{1} R_{1}^{T} X^{T}=0$ (because $\left.S_{1} R_{1}^{T}=\left(R_{1} S_{1}^{T}\right)^{T}=0\right)$, from which we conclude that $S_{1} S_{2}$ is singular.

The converse is proved in the same way, interchanging $R_{1}$ and $S_{1}, R_{2}$ and $S_{2}$. Thus $R_{1} R_{2}$ is singular if and only if $S_{1} S_{2}$ is singular.

Now suppose $R_{1} R_{2}^{T}$ and $S_{1} S_{2}$ are nonsingular. Let

$$
\begin{aligned}
M & =R_{2}\left(R_{1} R_{2}^{T}\right)^{-1} R_{1}+\left\{S_{2}^{T}\left(S_{1} S_{2}\right)^{-1} S_{1}\right\}^{T}-I_{n} \\
& =R_{2}\left(R_{1} R_{2}^{T}\right)^{-1} R_{1}+S_{1}^{T}\left(S_{2} S_{1}^{T}\right)^{-1} S_{2}-I_{n} .
\end{aligned}
$$

${ }^{1}$ This work arose from some matrix-theoretic questions posed by Dr. U. Fano.
We wish to prove that $M=0$. We have

$$
\begin{aligned}
R_{1} M & =R_{1} R_{2}\left(R_{1} R_{2}\right)^{-1} R_{1}+R_{1} S_{1}^{T}\left(S_{2} S_{1}^{T}\right)^{-1} S_{2}-R_{1} \\
& =R_{1}+0-R_{1} \\
& =0 .
\end{aligned}
$$

Similarly $S_{2} M=0$.

By Lemma 2, $R_{1} M=0$ implies that there exists an $N$ such that $M^{T}=N S_{1}$. Then

$$
0=\left(S_{2} M\right)^{T}=M^{T} S_{2}^{T}=N S_{1} S_{2}^{T}
$$

Since $S_{1} S_{2}^{T}$ is nonsingular, we conclude that $N=0$, whence $M=0$. This proves the theorem.

The following example shows that the characteristic roots (other than 0 ) of $R_{1} R_{2}^{T}$ and $S_{1} S_{2}^{T}$ need not have any relationship. Let

$$
\begin{aligned}
& R_{i}=A_{i}\left(I_{m} 0_{m, n-m}\right) \\
& S_{i}=B_{i}\left(0_{n-m, m} I_{n-m}\right)
\end{aligned} i=1,2
$$

where $A_{1}, A_{2}$ and $B_{1}, B_{2}$ are any nonsingular matrices of orders $m$ and $n-m$ respectively. Then $R_{1}, R_{2}$ $\epsilon \mathscr{R}_{m n}, \quad S_{1} \epsilon \mathcal{N}\left(R_{1}\right), \quad S_{2} \epsilon \mathcal{N}\left(R_{2}\right), \quad$ but $R_{1} R_{2}^{T}=A_{1} A_{2}^{T}$ and $S_{1} S_{2}^{T}=B_{1} B_{2}^{T}$. These latter two matrices are related only in the fact that both are nonsingular.

This is in direct contrast to the known result that a characteristic root of $F G$ ( $F$ and $G^{T}$ matrices of the same dimensions) is a characteristic root of $G F$, with the possible exception of a characteristic root equal to 0 ; i.e., $F G$ may be singular and $G F$ may be nonsingular, or vice-versa. 
Yet this result can be translated into one similar to our theorem. Since 1 is either a characteristic root of both $F G$ and $G F$ or neither, it follows that $I-F G$ is nonsingular if and only if $I-G F$ is nonsingular. If both are nonsingular it is easy to show that

$$
(I-F G)^{-1}-F(I-G F)^{-1} G=I_{p},
$$

where $p$ is the number of rows of $F$ (and columns of $G$ ).

Finally we note that if $R_{1}, R_{2}, S_{1}, S_{2}$ are as in the theorem and $A$ is nonsingular matrix of order $n$, then

$$
B=R_{1} A R_{2}^{T}, C=S_{2} A^{-1} S_{1}^{T}
$$

are nonsingular together in which case

$$
R_{2}^{T} B^{-1} R_{1} A+A^{-1} S_{1}^{T} C^{-1} S_{2}=I_{n} .
$$

This follows from the fact that $R_{1} A \epsilon \mathscr{R}_{m n}$ and $S_{1}\left(A^{T}\right)^{-1} \epsilon \mathcal{N}\left(R_{1} A\right)$.

(Paper 70B2-177) 